\title{
The Role of Customer Readiness in Customer Participation in Non-technology-based Service Delivery and Its Outcomes
}

\author{
Atieh Poushneh and Arturo Z. Vasquez-Parraga
}

\begin{abstract}
Whereas customer readiness has been found beneficial in the provision of tech-based services, its usefulness has not been thoroughly evaluated in the provision of non-tech-based services. Customer readiness seems to impact on customer participation in service delivery, and thus mediate the effects of many factors influencing such participation. This empirical chapter examines the role of customer readiness in customer participation in service delivery and its indirect effects on customer outcomes such as perceived service quality, customer satisfaction, and customer willingness to recommend. Because of its mediating role, customer readiness is also examined as the recipient of various antecedents of customer participation, such as previous experience, desire for control, need for interaction, product complexity, perceived risk, and customer organizational socialization. The results show that customer readiness has a significant impact on both customer participation and its outcomes, and that it effectively transfers the role of the four antecedents (previous experience, desire for control, perceived risk, and customer organizational socialization) to both customer participation and its outcomes.
\end{abstract}

\section{References available upon request.}

A. Poushneh $(\square) \bullet$ A.Z. Vasquez-Parraga

The University of Texas-Pan American, Edinburg, TX, USA

e-mail: aapoushneh@utpa.edu; avasquez@utpa.edu 